\title{
Copper-Catalyzed Asymmetric N-H Insertion Reactions: Couplings of Diazo Compounds with Carbamates to Generate $\alpha$-Amino Acids
}

\author{
Elaine C. Lee and Gregory C. Fu* \\ Department of Chemistry, Massachusetts Institute of Technology, \\ Cambridge, Massachusetts 02139
}

\section{Supporting Information}

General. All reactions were carried out in oven-dried glassware under an atmosphere of nitrogen. All chemicals were purchased from commercial suppliers and used as received, unless noted otherwise. $\mathrm{BocNH}_{2}$ (Fluka) was recrystallized from dichloromethane and pentane. 1,2-Dichloroethane (anhydrous; Fluka), $\mathrm{CuBr}$ (Strem), $\mathrm{AgSbF}_{6}(\mathrm{Strem})$, and $\mathrm{CbzNH}_{2}$ (Aldrich) were used as received. Bpy ${ }^{*}$ was prepared and resolved as previously described. ${ }^{1}$

HPLC analyses were carried out on an Agilent 1100 series system with Daicel Chiralpak ${ }^{\circledR}$ columns in hexane/isopropanol mixtures. GC analysis were carried out on an Agilent 6850 series system with a CP-Chirasil-Dex CB column; $140{ }^{\circ} \mathrm{C}(5.0 \mathrm{~min}), 1.0$ ${ }^{\circ} \mathrm{C} / \mathrm{min}$ to $160{ }^{\circ} \mathrm{C} ; 14.37 \mathrm{psi}$ He or on an Astec G-TA column; $140{ }^{\circ} \mathrm{C}$ (1.0 min), 1.0 ${ }^{\circ} \mathrm{C} / \mathrm{min}$ to $180^{\circ} \mathrm{C} ; 14.89 \mathrm{psi}$ He. Melting points were measured on a Hoover melting point apparatus and are uncorrected.

\section{Preparation of the Diazo Compounds}

The syntheses of the following diazo compounds have previously been described: tert-butyl $\alpha$-diazo- $\alpha$-phenylacetate [72410-67-4] and tert-butyl $\alpha$-diazo- $\alpha-4$ methoxyphenylacetate [76530-00-2].

The $\alpha$-aryl- $\alpha$-diazoesters were prepared via direct diazo transfer to the $\alpha$-aryl esters, as previously reported, using either tosyl azide or $p$-ABSA. ${ }^{2}$ The yields have not been optimized.<smiles>CCCCCC(=O)C(=N)c1ccccc1O</smiles>

The diazo ester was prepared from the ester $(3.10 \mathrm{~g}, 15.0 \mathrm{mmol})$, $\mathrm{MeCN}(53 \mathrm{~mL})$, DBU $(4.50 \mathrm{~mL}, 30.0 \mathrm{mmol})$, and $p$-ABSA (5.40 g, 22.5 mmol). Product: $1.02 \mathrm{~g}(29 \%)$; orange oil.

${ }^{1} \mathrm{H} \mathrm{NMR}\left(\mathrm{CDCl}_{3}, 300 \mathrm{MHz}\right) \delta$ 7.51-7.47 (m, 1H), 7.37-7.32 (m, 3H), $2.47(\mathrm{~s}, 3 \mathrm{H}), 1.63(\mathrm{~s}, 9 \mathrm{H})$;

${ }^{13} \mathrm{C} \mathrm{NMR}\left(\mathrm{CDCl}_{3}, 75.5 \mathrm{MHz}\right) \delta 165.5,137.6,130.93,130.89,128.7,126.5,124.8,81.9$, 28.6, 20.2; the resonance of the carbon that bears the diazo group was not detected;

IR (film) 2979, 2932, 2084, 1699, 1491, 1368, 1295, 1253, 1149, $1006 \mathrm{~cm}^{-1}$. 
<smiles>CCOC(=O)C(C#N)c1cccc(C)c1</smiles>

The diazo ester was prepared from the ester $(2.44 \mathrm{~g}, 11.8$ $\mathrm{mmol}), \mathrm{MeCN}(42 \mathrm{~mL}), \mathrm{DBU}(2.70 \mathrm{~mL}, 17.8 \mathrm{mmol})$, and $p$-ABSA (3.70 g, $15.4 \mathrm{mmol})$. Product: $1.33 \mathrm{~g}(47 \%)$; orange oil.

${ }^{1} \mathrm{H}$ NMR $\left(\mathrm{CDCl}_{3}, 300 \mathrm{MHz}\right) \delta$ 7.38-7.34 (m, 1H), 7.29-7.27 (m, 2H), 7.02-6.99 (m, 1H), $2.38(\mathrm{~s}, 3 \mathrm{H}), 1.58(\mathrm{~s}, 9 \mathrm{H})$;

${ }^{13} \mathrm{C} \mathrm{NMR}\left(\mathrm{CDCl}_{3}, 75.5 \mathrm{MHz}\right) \delta 164.9,138.8,128.9,126.6,126.1,124.8,121.2,82.1,28.6$, 21.8; the resonance of the carbon that bears the diazo group was not detected; IR (film) 2979, 2931, 2084, 1701, 1605, 1492, 1369, 1344, 1290, 1143, 1055, $779 \mathrm{~cm}^{-1}$.<smiles>CCCCOC(=O)C(=N)c1ccc(NC(=O)OC(C)(C)C)cc1</smiles>

The diazo ester was prepared from the ester $(1.87 \mathrm{~g}, 6.08$ $\mathrm{mmol}), \mathrm{MeCN}(32 \mathrm{~mL}), \mathrm{DBU}(1.36 \mathrm{~mL}, 9.23 \mathrm{mmol})$, and $p$ ABSA (1.90 g, $7.90 \mathrm{mmol})$. Product: $681 \mathrm{mg}$ (34\%); orange solid.

${ }^{1} \mathrm{H} \mathrm{NMR}\left(\mathrm{CDCl}_{3}, 500 \mathrm{MHz}\right) \delta 7.38$ (app s, 4H), 6.46 (s, 1H), 1.55 (s, 9H), 1.52 (s, 9H);

${ }^{13} \mathrm{C} \mathrm{NMR}\left(\mathrm{CDCl}_{3}, 126 \mathrm{MHz}\right) \delta 165.1,152.9,136.3,125.1,120.3,119.1,82.2(\mathrm{x} 2), 28.6$, 28.5; the resonance of the carbon that bears the diazo group was not detected; IR (film) 3055, 2987, 2306, 2086, 1693, 1521, 1422, 1266, 1150, 896, $744 \mathrm{~cm}^{-1}$.<smiles>CCCOC(=O)C(=N)c1ccc(Br)cc1</smiles>

The diazo ester was prepared from the ester $(1.67 \mathrm{~g}, 6.15$ $\mathrm{mmol}), \mathrm{MeCN}(28 \mathrm{~mL}), \mathrm{DBU}(1.38 \mathrm{~mL}, 9.23 \mathrm{mmol})$, and tosyl azide (1.54 g, $8.00 \mathrm{mmol})$. Product: $1.40 \mathrm{~g}(77 \%)$; orange solid.

${ }^{1} \mathrm{H} \mathrm{NMR}\left(\mathrm{CDCl}_{3}, 300 \mathrm{MHz}\right) \delta 7.48(\mathrm{~d}, J=9.0 \mathrm{~Hz}, 2 \mathrm{H}), 7.35(\mathrm{~d}, J$ $=8.7 \mathrm{~Hz}, 2 \mathrm{H}), 1.55(\mathrm{~s}, 9 \mathrm{H})$;

${ }^{13} \mathrm{C} \mathrm{NMR}\left(\mathrm{CDCl}_{3}, 75.5 \mathrm{MHz}\right) \delta 164.3,132.1,125.53,125.50,119.2,82.6,28.6$; the resonance of the carbon that bears the diazo group was not detected;

IR (film) 3055, 2984, 2088, 1696, 1491, 1347, 1266, 1148, 1003, 740, $705 \mathrm{~cm}^{-1}$.<smiles>CCCOC(=O)C(=N)c1ccc(C(F)(F)F)cc1</smiles>

The diazo ester was prepared from the ester $(3.46 \mathrm{~g}, 13.3$ $\mathrm{mmol}), \mathrm{MeCN}(60 \mathrm{~mL}), \mathrm{DBU}(2.90 \mathrm{~mL}, 20.0 \mathrm{mmol})$, and $p$-ABSA (4.20 g, $17.3 \mathrm{mmol})$. Product: $1.45 \mathrm{~g}(38 \%)$; orange solid.

${ }^{1} \mathrm{H} \mathrm{NMR}\left(\mathrm{CDCl}_{3}, 300 \mathrm{MHz}\right) \delta 7.60($ app s, $4 \mathrm{H}), 1.55$ (s, 9H);

${ }^{13} \mathrm{C} \mathrm{NMR}\left(\mathrm{CDCl}_{3}, 75.5 \mathrm{MHz}\right) \delta 163.9,130.9,127.4\left(\mathrm{q},{ }^{2} J_{\mathrm{CF}}=33.0 \mathrm{~Hz}\right), 125.9\left(\mathrm{q},{ }^{3} J_{\mathrm{CF}}=3.8\right.$ $\mathrm{Hz}), 123.6,122.5,82.9,28.5$; the resonance of the carbon that bears the diazo group was not detected;

IR (film) 3055, 2987, 2094, 1699, 1422, 1328, 1266, 1148, 896, 740, $705 \mathrm{~cm}^{-1}$.<smiles>CCCOC(=O)C(=N)c1ccc2ccccc2c1</smiles>

The diazo ester was prepared from the ester $(843 \mathrm{mg}, 3.48$ $\mathrm{mmol}), \mathrm{MeCN}(14 \mathrm{~mL}), \mathrm{DBU}(0.78 \mathrm{~mL}, 5.22 \mathrm{mmol})$, and tosyl azide (870 mg, $4.52 \mathrm{mmol})$. Product: $695 \mathrm{mg}$ (74\%); orange solid.

${ }^{1} \mathrm{H} \mathrm{NMR}\left(\mathrm{CDCl}_{3}, 300 \mathrm{MHz}\right) \delta 8.04(\mathrm{~s}, 1 \mathrm{H}), 7.86-7.79(\mathrm{~m}, 3 \mathrm{H})$, 
7.52-7.43 (m, 3H), 1.60 (s, 9H);

${ }^{13} \mathrm{C}$ NMR $\left(\mathrm{CDCl}_{3}, 75.5 \mathrm{MHz}\right) \delta 164.9,133.8,131.5,128.7,127.8,127.7,126.7,125.8$, $123.5,122.6,122.1,82.4,28.6$; the resonance of the carbon that bears the diazo group was not detected;

IR (film) 3055, 2987, 2306, 2088, 1696, 1422, 1266, 1148, 896, 739, $705 \mathrm{~cm}^{-1}$.<smiles>CCOC(=O)C(=N)c1ccc2c(c1)OCO2</smiles>

The diazo ester was prepared from the ester $(1.64 \mathrm{~g}, 6.94$ $\mathrm{mmol}), \mathrm{MeCN}(28 \mathrm{~mL}), \mathrm{DBU}(1.56 \mathrm{~mL}, 10.4 \mathrm{mmol})$, and tosyl azide (1.73 g, $9.02 \mathrm{mmol})$. Product: $489 \mathrm{mg}(27 \%)$; orange solid. ${ }^{1} \mathrm{H}$ NMR $\left(\mathrm{CDCl}_{3}, 300 \mathrm{MHz}\right) \delta$ 7.06-7.05 (m, 1H), 6.85-6.83 (m, $2 \mathrm{H}), 5.96(\mathrm{~s}, 2 \mathrm{H}), 1.54(\mathrm{~s}, 9 \mathrm{H})$;

${ }^{13} \mathrm{C} \mathrm{NMR}\left(\mathrm{CDCl}_{3}, 75.5 \mathrm{MHz}\right) \delta$ 165.1, 148.5, 146.0, 127.8, 117.9, 109.0, 105.9, 101.4, 82.2, 28.5); the resonance of the carbon that bears the diazo group was not detected;

IR (film) 3055, 2984, 2087, 1694, 1505, 1493, 1370, 1266, 1235, 1041, 740, $705 \mathrm{~cm}^{-1}$.<smiles>CCOC(=O)C(=N)c1ccsc1</smiles>

The diazo ester was prepared from the ester $(4.34 \mathrm{~g}, 21.9 \mathrm{mmol})$, $\mathrm{MeCN}$ (69 mL), DBU (4.90 mL, $32.8 \mathrm{mmol})$, and tosyl azide $(6.80 \mathrm{~g}$, $28.5 \mathrm{mmol})$. Product: $829 \mathrm{mg}$ (17\%); orange solid.

${ }^{1} \mathrm{H}$ NMR $\left(\mathrm{CDCl}_{3}, 300 \mathrm{MHz}\right) \delta$ 7.40-7.36 (m, 2H), 7.03-7.01 (m, 1H),

1.56 (s, 9H);

$\left.{ }^{13} \mathrm{C} \mathrm{NMR}\left(\mathrm{CDCl}_{3}, 75.5 \mathrm{MHz}\right) \delta 165.0,126.4,124.6,123.9,117.6,82.3,28.6\right)$; the resonance of the carbon that bears the diazo group was not detected;

IR (film) 3055, 2986, 2306, 2085, 1695, 1422, 1318, 1266, 1139, 896, 740, $705 \mathrm{~cm}^{-1}$. 


\section{Catalytic Asymmetric N-H Insertion Reactions}

All enantioselectivities and isolated yields that are reported in Tables 2 and 3 are the average of two experiments, one with (-)-bpy* and one with (+)-bpy*.

Typical Procedure for Table 2. Entry 1. In a glovebox, a solution of the catalyst was prepared by adding $\mathrm{CuBr}(4.7 \mathrm{mg}, 33 \mu \mathrm{mol}),(-)-\mathrm{bpy}^{*}(24.0 \mathrm{mg}, 37.5 \mu \mathrm{mol}), \mathrm{AgSbF}_{6}(9.7$ $\mathrm{mg}, 28 \mu \mathrm{mol})$, and 1,2-dichloroethane $(4.3 \mathrm{~mL})$ to a vial (\#1). The resulting mixture was stirred for $45 \mathrm{~min}$. In a separate vial (\#2), the diazo ester $(154 \mathrm{mg}, 0.704 \mathrm{mmol})$ and Boc $\mathrm{NH}_{2}(55.0 \mathrm{mg}, 0.469 \mathrm{mmol})$ were dissolved in 1,2-dichloroethane $(3.4 \mathrm{~mL})$, and this solution was added by syringe over $3 \mathrm{~min}$ to vial \#1 (vial \#2 was rinsed with 1,2dichloroethane $(0.1 \mathrm{~mL})$ ). The reaction mixture was stirred at room temperature for 30 min, and then the solvent was removed and the product was purified by flash chromatography.

When the reaction was set up outside of a glove box, a lower yield (64\%) and ee $(81 \%)$ were obtained (a single experiment).<smiles>CCOC(=O)C(N)c1ccccc1</smiles>

After chromatography on silica gel $\left(1 \% \mathrm{Et}_{2} \mathrm{O}\right.$ in $\left.\mathrm{CH}_{2} \mathrm{Cl}_{2}\right)$, the desired compound was isolated as a white solid: run 1, $105 \mathrm{mg}(73 \%$; $93 \%$ ee); run 2, $110 \mathrm{mg}(76 \%$; $95 \%$ ee). The ee was determined on a CP-Chirasil-Dex CB column with $\mathrm{t}_{\mathrm{r}}$ (major) 21.6 min, $\mathrm{t}_{\mathrm{r}}($ minor) $22.0 \mathrm{~min}$. mp: $115-116^{\circ} \mathrm{C}$; $[\alpha]_{D}^{20}=-120\left(\mathrm{c}=0.95, \mathrm{CH}_{2} \mathrm{Cl}_{2}\right) ; 95 \%$ ee, from $(+)-\mathrm{bpy}^{*}$; ${ }^{1} \mathrm{H}$ NMR $\left(\mathrm{CDCl}_{3}, 500 \mathrm{MHz}\right) \delta$ 7.29-7.21 (m, 5H), $5.53(\mathrm{~d}, J=6.5 \mathrm{~Hz}, 1 \mathrm{H}), 5.13(\mathrm{~d}, J=$ $7.5 \mathrm{~Hz}, 1 \mathrm{H}), 1.36$ (s, 9H), 1.31 (s, 9H);

${ }^{13} \mathrm{C} \mathrm{NMR}\left(\mathrm{CDCl}_{3}, 126 \mathrm{MHz}\right) \delta$ 170.4, 155.0, 137.0, 128.8, 128.2, 127.1, 82.5, 78.0, 58.2, 28.5, 27.9;

IR (film) 3055, 2987, 2306, 1422, 1266, 896, 740, $705 \mathrm{~cm}^{-1}$; HRMS (ESI) calcd for $\mathrm{C}_{17} \mathrm{H}_{25} \mathrm{NNaO}_{4}\left(\mathrm{M}+\mathrm{Na}^{+}\right) 330.1676$, found 330.1679.<smiles>CCOC(=O)C(NCc1ccccc1)c1ccccc1OC</smiles>

Table 2, entry 2. The compound was prepared with (-)-bpy* from the diazo ester $(119 \mathrm{mg}, 0.512 \mathrm{mmol})$ and $\mathrm{BocNH}_{2}(40.0 \mathrm{mg}, 0.341$ $\mathrm{mmol})$. After chromatography on silica gel $\left(1 \% \mathrm{Et}_{2} \mathrm{O}\right.$ in $\left.\mathrm{CH}_{2} \mathrm{Cl}_{2}\right)$, the desired compound was isolated as a colorless oil: run 1, 74.0 mg (68\%; 80\% ee); run 2, $81.0 \mathrm{mg}$ (74\%; $81 \%$ ee). The ee was determined on an Astec G-TA column with $\mathrm{t}_{\mathrm{r}}$ (major) $37.1 \mathrm{~min}, \mathrm{t}_{\mathrm{r}}($ minor) $37.6 \mathrm{~min}$.

$[\alpha]_{D}^{20}=+86\left(\mathrm{c}=1.25, \mathrm{CH}_{2} \mathrm{Cl}_{2}\right) ; 80 \%$ ee, from $(-)-\mathrm{bpy}^{*}$;

${ }^{1} \mathrm{H}$ NMR $\left(\mathrm{CDCl}_{3}, 500 \mathrm{MHz}\right) \delta$ 7.27-7.16 $(\mathrm{m}, 4 \mathrm{H}), 5.52(\mathrm{~d}, J=7.0 \mathrm{~Hz}, 1 \mathrm{H}), 5.44(\mathrm{~d}, J=$ $7.5 \mathrm{~Hz}, 1 \mathrm{H}), 1.43$ (s, 9H), 1.37 (s, 9H); 
${ }^{13} \mathrm{C} \mathrm{NMR}\left(\mathrm{CDCl}_{3}, 75.5 \mathrm{MHz}\right) \delta 170.9,155.1,136.8,136.5,130.9,128.1,126.4,126.1,82.3$, $79.9,54.7,28.5,28.0,19.7$;

IR (film) 3055, 2985, 2306, 1712, 1492, 1369, 1266, 1154, 896, 740, $705 \mathrm{~cm}^{-1}$;

HRMS (ESI) calcd for $\mathrm{C}_{18} \mathrm{H}_{27} \mathrm{NNaO}_{4}\left(\mathrm{M}+\mathrm{Na}^{+}\right)$344.1832, found 344.1838.<smiles>CCCCC(=O)OC(=O)C(N)c1cccc(C)c1</smiles>

Table 2, entry 3. The compound was prepared with (-)-bpy* from the diazo ester $(163 \mathrm{mg}, 0.704 \mathrm{mmol})$ and $\mathrm{BocNH}_{2}(55.0 \mathrm{mg}$, $0.469 \mathrm{mmol})$. After chromatography on silica gel $\left(1 \% \mathrm{Et}_{2} \mathrm{O}\right.$ in

$\left.\mathrm{CH}_{2} \mathrm{Cl}_{2}\right)$, the desired compound was isolated as a colorless oil: run $1,114 \mathrm{mg}(76 \% ; 88 \%$ ee); run 2 (with $0.427 \mathrm{mmol}$ of $\left.\mathrm{BocNH}_{2}\right), 100 \mathrm{mg}(73 \%$; 87\% ee). The ee was determined on an AD-H column (hexanes/iso-propanol 90:10, flow $1.0 \mathrm{~mL} / \mathrm{min}$ ) with $\mathrm{t}_{\mathrm{r}}(\mathrm{minor}) 6.5$ min, $\mathrm{t}_{\mathrm{r}}$ (major) $10.4 \mathrm{~min}$.

$[\alpha]^{20}{ }_{D}=+88\left(\mathrm{c}=1.50, \mathrm{CH}_{2} \mathrm{Cl}_{2}\right) ; 88 \%$ ee, from (-)-bpy*;

${ }^{1} \mathrm{H}$ NMR $\left(\mathrm{CDCl}_{3}, 500 \mathrm{MHz}\right) \delta 7.22(\mathrm{dd}, J=15,7.5 \mathrm{~Hz}, 1 \mathrm{H}), 7.16-7.09(\mathrm{~m}, 3 \mathrm{H}), 5.55(\mathrm{~d}, J$ $=7.5 \mathrm{~Hz}, 1 \mathrm{H}), 5.16(\mathrm{~d}, J=7.5 \mathrm{~Hz}, 1 \mathrm{H}), 2.34(\mathrm{~s}, 3 \mathrm{H}), 1.44(\mathrm{~s}, 9 \mathrm{H}), 1.40(\mathrm{~s}, 9 \mathrm{H})$;

${ }^{13} \mathrm{C} \mathrm{NMR}\left(\mathrm{CDCl}_{3}, 126 \mathrm{MHz}\right) \delta 170.5,155.0,138.5,137.7,129.0,128.7,127.9,124.1,82.4$, 80.0, 58.2, 28.5, 28.0, 21.6;

IR (film) 3055, 2984, 2306, 1712, 1494, 1266, 1155, 1052, 740, $705 \mathrm{~cm}^{-1}$;

HRMS (ESI) calcd for $\mathrm{C}_{18} \mathrm{H}_{27} \mathrm{NNaO}_{4}\left(\mathrm{M}+\mathrm{Na}^{+}\right)$344.1832, found 344.1833.<smiles>CCCCC(=O)C(=O)N[C@@H](c1ccccc1)c1ccc(OC)cc1</smiles>

Table 2, entry 4. The compound was prepared with (-)$\mathrm{bpy}^{*}$ from the diazo ester $(159 \mathrm{mg}, 0.640 \mathrm{mmol})$ and $\mathrm{BocNH}_{2}$ (50.0 $\mathrm{mg}, 0.427 \mathrm{mmol})$. After chromatography on silica gel $(1 \%$ $\mathrm{Et}_{2} \mathrm{O}$ in $\mathrm{CH}_{2} \mathrm{Cl}_{2}$ ), the desired compound was isolated as a colorless oil: run 1, $89.0 \mathrm{mg}$ (62\%; $94 \%$ ee); run 2, $86.0 \mathrm{mg}(60 \%$; $95 \%$ ee). The ee was determined on an AD-H column (hexanes/iso-propanol 90:10, flow $1.0 \mathrm{~mL} / \mathrm{min}$ ) with $\mathrm{t}_{\mathrm{r}}$ (minor) $9.4 \mathrm{~min}, \mathrm{t}_{\mathrm{r}}($ major) $11.1 \mathrm{~min}$.

$[\alpha]_{D}^{20}=+106\left(\mathrm{c}=1.20, \mathrm{CH}_{2} \mathrm{Cl}_{2}\right) ; 94 \%$ ee, from $(-)-\mathrm{bpy}^{*}$;

${ }^{1} \mathrm{H}$ NMR $\left(\mathrm{CDCl}_{3}, 500 \mathrm{MHz}\right) \delta 7.28(\mathrm{~d}, J=8.0 \mathrm{~Hz}, 2 \mathrm{H}), 6.87(\mathrm{~d}, J=9.0 \mathrm{~Hz}, 2 \mathrm{H}), 5.56(\mathrm{~d}$, $J=7.0 \mathrm{~Hz}, 1 \mathrm{H}), 5.14(\mathrm{~d}, J=7.5 \mathrm{~Hz}, 1 \mathrm{H}), 3.78(\mathrm{~s}, 3 \mathrm{H}), 1.44(\mathrm{~s}, 9 \mathrm{H}), 1.40(\mathrm{~s}, 9 \mathrm{H})$;

${ }^{13} \mathrm{C} \mathrm{NMR}\left(\mathrm{CDCl}_{3}, 126 \mathrm{MHz}\right) \delta$ 170.6, 159.4, 154.9, 130.0, 128.3, 114.3, 82.3, 79.9, 57.6, $55.3,28.5,27.9$;

IR (film) 3055, 2987, 2306, 1422, 1266, 896, 740, $705 \mathrm{~cm}^{-1}$;

HRMS (ESI) calcd for $\mathrm{C}_{18} \mathrm{H}_{27} \mathrm{NNaO}_{5}\left(\mathrm{M}+\mathrm{Na}^{+}\right) 360.1781$, found 360.1772 .

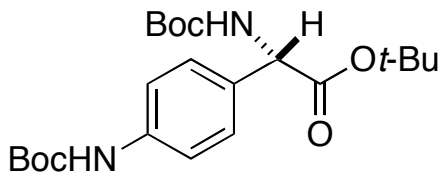

Table 2, entry 5. The compound was prepared with (-)$\mathrm{bpy}^{*}$ from the diazo ester $\left(171 \mathrm{mg}, 0.512 \mathrm{mmol}\right.$ ) and $\mathrm{BocNH}_{2}$ ( $40.0 \mathrm{mg}, 0.341 \mathrm{mmol}$ ). After chromatography on silica gel ( $5 \% \mathrm{Et}_{2} \mathrm{O}$ in $\mathrm{CH}_{2} \mathrm{Cl}_{2}$ ), the desired compound was isolated as a white solid: run 1, $112 \mathrm{mg}$ (78\%; 89\% ee); run 2, $109 \mathrm{mg}$ (76\%; 92\% ee). The ee was 
determined on an AS-H column (hexanes/iso-propanol 95:5, flow $1.0 \mathrm{~mL} / \mathrm{min}$ ) with $\mathrm{t}_{\mathrm{r}}$ (minor) $11.6 \mathrm{~min}, \mathrm{t}_{\mathrm{r}}$ (major) $15.9 \mathrm{~min}$.

$\mathrm{mp}: 174-175^{\circ} \mathrm{C}$;

$[\alpha]^{20}{ }_{D}=-80\left(\mathrm{c}=1.20, \mathrm{CH}_{2} \mathrm{Cl}_{2}\right) ; 92 \%$ ee, from $(+)-\mathrm{bpy}^{*}$;

${ }^{1} \mathrm{H}$ NMR $\left(\mathrm{CDCl}_{3}, 500 \mathrm{MHz}\right) \delta 7.34(\mathrm{~d}, J=8.0 \mathrm{~Hz}, 2 \mathrm{H}), 7.26(\mathrm{~d}, J=8.5 \mathrm{~Hz}, 2 \mathrm{H}), 6.69(\mathrm{~s}$, $1 \mathrm{H}), 5.57(\mathrm{~d}, J=7.0 \mathrm{~Hz}, 1 \mathrm{H}), 5.14(\mathrm{~d}, J=7.5 \mathrm{~Hz}, 1 \mathrm{H}), 1.51(\mathrm{~s}, 9 \mathrm{H}), 1.43(\mathrm{~s}, 9 \mathrm{H}), 1.38(\mathrm{~s}, 9 \mathrm{H})$;

${ }^{13} \mathrm{C} \mathrm{NMR}\left(\mathrm{CDCl}_{3}, 75.5 \mathrm{MHz}\right) \delta 170.4,155.0,152.9,138.4,132.3,129.1,127.8,118.7,82.5$, 80.0, 57.7, 28.5, 28.0;

IR (film) 4453, 4197, 3945, 3055, 2987, 2306, 1728, 1422, 1266, 1156, 896, $740 \mathrm{~cm}^{-1}$;

HRMS (ESI) calcd for $\mathrm{C}_{22} \mathrm{H}_{34} \mathrm{~N}_{2} \mathrm{NaO}_{6}\left(\mathrm{M}+\mathrm{Na}^{+}\right) 445.2309$, found 445.2313.

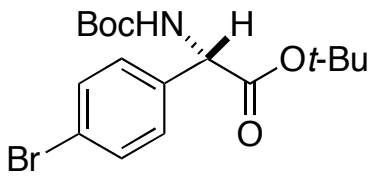

Table 2, entry 6. The compound was prepared with (-)-bpy* from the diazo ester $(152 \mathrm{mg}, 0.512 \mathrm{mmol})$ and $\mathrm{BocNH}_{2}(40.0 \mathrm{mg}$, $0.341 \mathrm{mmol})$. After chromatography on silica gel $\left(2 \% \mathrm{Et}_{2} \mathrm{O}\right.$ in $\mathrm{CH}_{2} \mathrm{Cl}_{2}$ ), the desired compound was isolated as a colorless oil: run $1,113 \mathrm{mg}$ ( $86 \%$; 85\% ee); run 2, $113 \mathrm{mg}(86 \% ; 85 \%$ ee). The ee was determined on an AD-H column (hexanes/iso-propanol 95:5, flow $1.0 \mathrm{~mL} / \mathrm{min}$ ) with $\mathrm{t}_{\mathrm{r}}$ (minor) $15.8 \mathrm{~min}$, $\mathrm{t}_{\mathrm{r}}($ major) $17.0 \mathrm{~min}$.

$[\alpha]^{20}=+72\left(\mathrm{c}=1.65, \mathrm{CH}_{2} \mathrm{Cl}_{2}\right) ; 85 \%$ ee, from $(-)-\mathrm{bpy}^{*}$;

${ }^{1} \mathrm{H}$ NMR $\left(\mathrm{CDCl}_{3}, 300 \mathrm{MHz}\right) \delta 7.46(\mathrm{~d}, J=8.4 \mathrm{~Hz}, 2 \mathrm{H}), 7.24(\mathrm{~d}, J=8.4 \mathrm{~Hz}, 2 \mathrm{H}), 5.65(\mathrm{~d}$, $J=6.9 \mathrm{~Hz}, 1 \mathrm{H}), 5.15(\mathrm{~d}, J=7.2 \mathrm{~Hz}, 1 \mathrm{H}), 1.42(\mathrm{~s}, 9 \mathrm{H}), 1.38(\mathrm{~s}, 9 \mathrm{H})$;

${ }^{13} \mathrm{C} \mathrm{NMR}\left(\mathrm{CDCl}_{3}, 75.5 \mathrm{MHz}\right) \delta 169.8,154.9,137.2,131.9,128.8,122.2,83.0,80.3,57.7$, 28.5, 27.9;

IR (film) 3055, 2987, 2306, 1712, 1422, 1266, 1156, 896, 706, $736 \mathrm{~cm}^{-1}$;

HRMS (ESI) calcd for $\mathrm{C}_{17} \mathrm{H}_{24} \mathrm{BrNNaO}_{4}\left(\mathrm{M}+\mathrm{Na}^{+}\right)$408.0781, found 408.0789.<smiles>CCOC(=O)C(N)c1ccc(C(F)(F)F)cc1</smiles>

Table 2, entry 7. The compound was prepared with (-)-bpy* from the diazo ester $(146 \mathrm{mg}, 0.512 \mathrm{mmol})$ and $\mathrm{BocNH}_{2}(40.0 \mathrm{mg}$, $0.341 \mathrm{mmol})$. After chromatography on silica gel $\left(1 \% \mathrm{Et}_{2} \mathrm{O}\right.$ in $\mathrm{CH}_{2} \mathrm{Cl}_{2}$ ), the desired compound was isolated as a colorless oil:

run $1,113 \mathrm{mg}(88 \% ; 85 \%$ ee); run 2, $115 \mathrm{mg}$ (90\%; 84\% ee). The ee was determined on an AD-H column (hexanes/iso-propanol 99:1, flow $1.0 \mathrm{~mL} / \mathrm{min}$ ) with $\mathrm{t}_{\mathrm{r}}$ (major) $17.2 \mathrm{~min}$, $\mathrm{t}_{\mathrm{r}}$ (minor) $19.1 \mathrm{~min}$.

$[\alpha]^{20}{ }_{D}=+77\left(\mathrm{c}=1.25, \mathrm{CH}_{2} \mathrm{Cl}_{2}\right) ; 85 \%$ ee, from $(-)-\mathrm{bpy}^{*}$;

${ }^{1} \mathrm{H} \mathrm{NMR}\left(\mathrm{CDCl}_{3}, 500 \mathrm{MHz}\right) \delta 7.60(\mathrm{~d}, J=8.0 \mathrm{~Hz}, 2 \mathrm{H}), 7.49(\mathrm{~d}, J=8.0 \mathrm{~Hz}, 2 \mathrm{H}), 5.76(\mathrm{~d}$, $J=7.0 \mathrm{~Hz}, 1 \mathrm{H}), 5.26(\mathrm{~d}, J=7.0 \mathrm{~Hz}, 1 \mathrm{H}), 1.42(\mathrm{~s}, 9 \mathrm{H}), 1.39(\mathrm{~s}, 9 \mathrm{H})$;

${ }^{13} \mathrm{C} \mathrm{NMR}\left(\mathrm{CDCl}_{3}, 126 \mathrm{MHz}\right) \delta 169.5,154.9,142.2,130.4\left(\mathrm{q},{ }^{2} J_{\mathrm{CF}}=32.2 \mathrm{~Hz}\right), 127.4,125.8$ $\left(\mathrm{q},{ }^{3} J_{\mathrm{CF}}=3.4 \mathrm{~Hz}\right), 124.2\left(\mathrm{q}, J_{\mathrm{CF}}=272 \mathrm{~Hz}\right), 83.2,80.4,57.9,28.5,27.9$;

IR (film) 3055, 2987, 2306, 1713, 1422, 1326, 1266, 1156, 896, 736, $706 \mathrm{~cm}^{-1}$; HRMS (ESI) calcd for $\mathrm{C}_{18} \mathrm{H}_{24} \mathrm{~F}_{3} \mathrm{NNaO}_{4}\left(\mathrm{M}+\mathrm{Na}^{+}\right)$398.1550, found 398.1559. 
<smiles>CCCCOC(=O)[C@H](N)c1ccc2ccccc2c1</smiles>

Table 2, entry 8. The compound was prepared with (-)-bpy* from the diazo ester $(155 \mathrm{mg}, 0.578 \mathrm{mmol})$ and $\mathrm{BocNH}_{2}(45.1 \mathrm{mg}$, $0.385 \mathrm{mmol})$. After chromatography on silica gel $\left(1 \% \mathrm{Et}_{2} \mathrm{O}\right.$ in $\left.\mathrm{CH}_{2} \mathrm{Cl}_{2}\right)$, the desired compound was isolated as a white solid: run 1, $96.0 \mathrm{mg}(70 \% ; 89 \%$ ee); run 2, $103 \mathrm{mg}$ (75\%; 92\% ee). The ee was determined on an AD-H column (hexanes / iso-propanol 90:10, flow $1.0 \mathrm{~mL} / \mathrm{min}$ ) with $\mathrm{t}_{\mathrm{r}}$ (minor) $7.4 \mathrm{~min}, \mathrm{t}_{\mathrm{r}}$ (major) 10.3 min.

mp: $172-173^{\circ} \mathrm{C}$;

$[\alpha]^{20}=+106\left(\mathrm{c}=1.20, \mathrm{CH}_{2} \mathrm{Cl}_{2}\right) ; 89 \%$ ee, from $(-)-\mathrm{bpy}^{*}$;

${ }^{1} \mathrm{H} \mathrm{NMR}\left(\mathrm{CDCl}_{3}, 500 \mathrm{MHz}\right) \delta$ 7.85-7.83 (m, 4H), 7.50-7.47 (m, 3H), 5.73 (d, J=7.0 Hz, $1 \mathrm{H}), 5.38$ (d, J=7.5 Hz, 1H), 1.45 (s, 9H), 1.39 (s, 9H);

${ }^{13} \mathrm{C} \mathrm{NMR}\left(\mathrm{CDCl}_{3}, 126 \mathrm{MHz}\right) \delta 170.4,155.0,135.4,133.5,133.2,128.7,128.3,127.9$ (x2), 126.4 (x2), 124.9, 82.7, 80.1, 58.4, 28.5, 28.0;

IR (film) 3055, 2986, 2306, 1712, 1422, 1266, 1155, 896, 739, $705 \mathrm{~cm}^{-1}$;

HRMS (ESI) calcd for $\mathrm{C}_{21} \mathrm{H}_{27} \mathrm{NNaO}_{4}\left(\mathrm{M}+\mathrm{Na}^{+}\right)$380.1832, found 380.1831.<smiles>CCCOC(=O)[C@H](N)c1ccc2c(c1)OCO2</smiles>

Table 2, entry 9. The compound was prepared with (-)-bpy* from the diazo ester $(120 \mathrm{mg}, 0.458 \mathrm{mmol})$ and $\mathrm{BocNH}_{2}(35.7 \mathrm{mg}$, $0.305 \mathrm{mmol})$. After chromatography on silica gel $\left(2 \% \mathrm{Et}_{2} \mathrm{O}\right.$ in $\left.\mathrm{CH}_{2} \mathrm{Cl}_{2}\right)$, the desired compound was isolated as a colorless oil: run 1, $80.0 \mathrm{mg}(75 \% ; 89 \%$ ee); run 2, $77.0 \mathrm{mg}(72 \% ; 91 \%$ ee). The ee was determined on an AD-H column (hexanes/iso-propanol 90:10, flow $1.0 \mathrm{~mL} / \mathrm{min}$ ) with $\mathrm{t}_{\mathrm{r}}$ (minor) $9.2 \mathrm{~min}, \mathrm{t}_{\mathrm{r}}$ (major) 13.2 min.

$[\alpha]^{20}{ }_{D}=+80\left(\mathrm{c}=1.80, \mathrm{CH}_{2} \mathrm{Cl}_{2}\right) ; 89 \%$ ee, from $(-)$-bpy*;

${ }^{1} \mathrm{H} \mathrm{NMR}\left(\mathrm{CDCl}_{3}, 300 \mathrm{MHz}\right) \delta$ 6.83-6.75 (m, 3H), $5.95(\mathrm{~s}, 2 \mathrm{H}), 5.56(\mathrm{~d}, J=6.6 \mathrm{~Hz}, 1 \mathrm{H})$, $5.09(\mathrm{~d}, J=7.5 \mathrm{~Hz}, 1 \mathrm{H}), 1.43(\mathrm{~s}, 9 \mathrm{H}), 1.40(\mathrm{~s}, 9 \mathrm{H})$;

${ }^{13} \mathrm{C} \mathrm{NMR}\left(\mathrm{CDCl}_{3}, 75.5 \mathrm{MHz}\right) \delta 170.4,154.9,148.0,147.6,131.8,120.7,108.6,107.6$, $101.3,82.6,80.1,57.9,28.5,27.9$;

IR (film) 3055, 2987, 2306, 1715, 1422, 1266, 1155, 896, 740, $705 \mathrm{~cm}^{-1}$;

HRMS (ESI) calcd for $\mathrm{C}_{18} \mathrm{H}_{25} \mathrm{NNaO}_{6}\left(\mathrm{M}+\mathrm{Na}^{+}\right) 374.1574$, found 374.1572.

Table 2, entry 10. The compound was prepared with (-)-bpy*<smiles>CCOC(=O)C(N)c1ccsc1</smiles>
from the diazo ester $(172 \mathrm{mg}, 0.768 \mathrm{mmol})$ and $\mathrm{BocNH}_{2}(60.0 \mathrm{mg}$, $0.512 \mathrm{mmol})$. After chromatography on silica gel $\left(1 \% \mathrm{Et}_{2} \mathrm{O}\right.$ in $\mathrm{CH}_{2} \mathrm{Cl}_{2}$ ), the desired compound was isolated as a yellow oil: run 1 , $84.0 \mathrm{mg}$ ( $51 \%$; $82 \%$ ee); run $2,77.0 \mathrm{mg}(46 \% ; 77 \%$ ee). The ee was determined on an IA column (hexanes/iso-propanol 95:5, flow $1.0 \mathrm{~mL} / \mathrm{min}$ ) with $\mathrm{t}_{\mathrm{r}}$ (minor) $11.3 \mathrm{~min}$, $\mathrm{t}_{\mathrm{r}}$ (major) $14.3 \mathrm{~min}$.

$$
[\alpha]_{D}^{20}=-52\left(\mathrm{c}=1.70, \mathrm{CH}_{2} \mathrm{Cl}_{2}\right) ; 77 \% \text { ee, from }(+)-\mathrm{bpy}^{*} ;
$$


${ }^{1} \mathrm{H} \mathrm{NMR}\left(\mathrm{CDCl}_{3}, 300 \mathrm{MHz}\right)$ 8 7.31-7.28 (m, 1H), 7.24-7.23 (m, 1H), $7.08(\mathrm{dd}, J=3.6,1.2$ $\mathrm{Hz}, 1 \mathrm{H}), 5.46(\mathrm{~d}, J=7.5 \mathrm{~Hz}, 1 \mathrm{H}), 5.32(\mathrm{~d}, J=8.4 \mathrm{~Hz}, 1 \mathrm{H}), 1.45(\mathrm{~s}, 9 \mathrm{H}), 1.44(\mathrm{~s}, 9 \mathrm{H})$;

${ }^{13} \mathrm{C} \mathrm{NMR}\left(\mathrm{CDCl}_{3}, 75.5 \mathrm{MHz}\right) \delta$ 170.1, 153.6, 138.2, 126.5, 126.4, 122.5, 82.7, 80.2, 54.3, 28.5, 28.1;

IR (film) 3430, 3054, 2983, 2306, 1714, 1493, 1369, 1265, 1154, 1055, 744, $705 \mathrm{~cm}^{-1}$; HRMS (ESI) calcd for $\mathrm{C}_{15} \mathrm{H}_{23} \mathrm{NNaO}_{4} \mathrm{~S}\left(\mathrm{M}+\mathrm{Na}^{+}\right) 336.1240$, found 336.1246.

Typical Procedure for Table 3. Entry 1. In a glovebox, a solution of the catalyst was prepared by mixing $\mathrm{CuBr}(3.8 \mathrm{mg}, 27 \mu \mathrm{mol}),(-)-\mathrm{bpy}^{*}(19.6 \mathrm{mg}, 30.6 \mu \mathrm{mol}), \mathrm{AgSbF}_{6}(7.9$ $\mathrm{mg}, 23 \mu \mathrm{mol})$, and 1,2-dichloroethane $(26 \mathrm{~mL})$ in a vial (\#1). This mixture was stirred for $45 \mathrm{~min}$, and then a portion $(8.6 \mathrm{~mL})$ was placed into a separate vial (\#2). In another vial (\#3), the diazo ester $(83.4 \mathrm{mg}, 0.382 \mathrm{mmol})$ and $\mathrm{CbzNH}_{2}(60.0 \mathrm{mg}, 0.382 \mathrm{mmol})$ were dissolved in 1,2-dichloroethane $(6.0 \mathrm{~mL})$. This solution was added by syringe over 3 min to vial \#1 (additional 1,2-dichloroethane $(0.1 \mathrm{~mL})$ was used to rinse vial \#3, and this was added to vial \#1). The reaction mixture was allowed to stir for $5 \mathrm{~min}$, and then the remainder of the catalyst (vial \#2) was added in one portion. Finally, the remainder of the diazo ester $(41.7 \mathrm{mg}, 0.191 \mathrm{mmol})$ in 1,2-dichloroethane $(6.0 \mathrm{~mL})$ was added dropwise over $0.5 \mathrm{~min}$ to the reaction mixture. The mixture was stirred at room temperature for $30 \mathrm{~min}$, concentrated to dryness, and purified by flash chromatography.

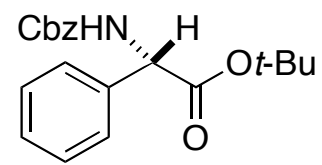

After chromatography on silica gel $\left(0.5 \% \mathrm{Et}_{2} \mathrm{O}\right.$ in $\left.\mathrm{CH}_{2} \mathrm{Cl}_{2}\right)$, the desired compound was isolated as a colorless oil: run $1,96.0 \mathrm{mg}$ (74\%; 94\% ee); run 2, $110 \mathrm{mg}$ (77\%; 95\% ee). The ee was determined on an AD-H column (hexanes/iso-propanol 95:5, flow $1.0 \mathrm{~mL} / \mathrm{min}$ ) with $\mathrm{t}_{\mathrm{r}}$ (minor) 19.5 $\min , \mathrm{t}_{\mathrm{r}}($ major $) 25.5 \mathrm{~min}$.

$[\alpha]^{20}{ }_{D}=-71\left(\mathrm{c}=2.35, \mathrm{CH}_{2} \mathrm{Cl}_{2}\right) ; 94 \%$ ee, from (+)-bpy*;

${ }^{1} \mathrm{H} \mathrm{NMR}\left(\mathrm{CDCl}_{3}, 300 \mathrm{MHz}\right) \delta 7.36($ br app s, $10 \mathrm{H}), 5.89(\mathrm{~d}, J=6.9 \mathrm{~Hz}, 1 \mathrm{H}), 5.28(\mathrm{~d}, J=$ $7.5 \mathrm{~Hz}, 1 \mathrm{H}), 5.11(\mathrm{dt}, J=8.7 \mathrm{~Hz}, 12 \mathrm{~Hz}, 2 \mathrm{H}), 1.40(\mathrm{~s}, 9 \mathrm{H})$;

${ }^{13} \mathrm{C} \mathrm{NMR}\left(\mathrm{CDCl}_{3}, 75.5 \mathrm{MHz}\right) \delta 175.7,155.5,137.5,136.4,128.9,128.7,128.4,128.3$, 127.1, 82.8, 67.1, 58.5, 27.9;

IR (film) 3424, 3055, 2984, 2306, 1722, 1498, 1266, 1153, 1051, 739, $704 \mathrm{~cm}^{-1}$; HRMS (ESI) calcd for $\mathrm{C}_{20} \mathrm{H}_{23} \mathrm{NNaO}_{4}\left(\mathrm{M}+\mathrm{Na}^{+}\right) 364.1519$, found 364.1520 .

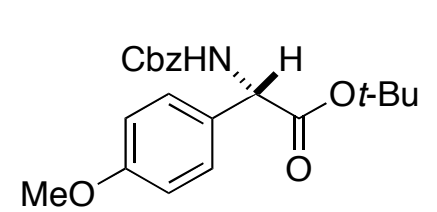

Table 3, entry 2. The compound was prepared with (-)bpy* from the diazo ester $(130 \mathrm{mg}, 0.525 \mathrm{mmol})$ and $\mathrm{CbzNH}_{2}$ $(55.0 \mathrm{mg}, 0.350 \mathrm{mmol})$. After chromatography on silica gel $\left(100 \% \mathrm{CH}_{2} \mathrm{Cl}_{2}\right)$, the desired compound was isolated as a colorless oil: run 1, $62 \mathrm{mg}$ ( $48 \%$; 90\% ee); run 2, $110 \mathrm{mg}$ (49\%; 90\% ee). The ee was determined on an AD-H column (hexanes/iso-propanol 90:10, flow $1.0 \mathrm{~mL} / \mathrm{min}$ ) with $\mathrm{t}_{\mathrm{r}}$ (minor) $22.1 \mathrm{~min}, \mathrm{t}_{\mathrm{r}}$ (major) $24.5 \mathrm{~min}$. 
$[\alpha]^{20}{ }_{D}=-92\left(\mathrm{c}=1.30, \mathrm{CH}_{2} \mathrm{Cl}_{2}\right) ; 90 \%$ ee, from $(+)-\mathrm{bpy}^{*}$;

${ }^{1} \mathrm{H}$ NMR $\left(\mathrm{CDCl}_{3}, 300 \mathrm{MHz}\right) \delta 7.27(\mathrm{br}$ app s, $5 \mathrm{H}), 7.21(\mathrm{~d}, J=8.7 \mathrm{~Hz}, 2 \mathrm{H}), 6.79(\mathrm{~d}, J=$ $8.7 \mathrm{~Hz}, 2 \mathrm{H}), 5.76(\mathrm{~d}, J=7.2 \mathrm{~Hz}, 1 \mathrm{H}), 5.12(\mathrm{~d}, J=7.5 \mathrm{~Hz}, 1 \mathrm{H}), 5.02(\mathrm{dt}, J=7.8 \mathrm{~Hz}, 12 \mathrm{~Hz}$, $2 \mathrm{H}), 3.72(\mathrm{~s}, 3 \mathrm{H}), 1.32(\mathrm{~s}, 9 \mathrm{H})$;

${ }^{13} \mathrm{C} \mathrm{NMR}\left(\mathrm{CDCl}_{3}, 75.5 \mathrm{MHz}\right) \delta 170.2,159.6,155.5,136.4,129.7,128.7,128.4,114.3,82.6$, 67.1, 58.0, 55.4, 28.0;

IR (film) 3055, 2987, 2306, 1719, 1512, 1421, 1266, 1154, 896, 740, $705 \mathrm{~cm}^{-1}$;

HRMS (ESI) calcd for $\mathrm{C}_{21} \mathrm{H}_{25} \mathrm{NNaO}_{5}\left(\mathrm{M}+\mathrm{Na}^{+}\right)$394.1625, found 394.1619.<smiles>CCCCC(=O)OC(=O)[C@H](NC(=O)c1ccc(C(F)(F)F)cc1)c1ccccc1</smiles>

Table 3, entry 3. The compound was prepared with (-)-bpy* from the diazo ester $(137 \mathrm{mg}, 0.477 \mathrm{mmol})$ and $\mathrm{CbzNH}_{2}(50.0$ $\mathrm{mg}, 0.318 \mathrm{mmol})$. After chromatography on silica gel $\left(1.0 \% \mathrm{Et}_{2} \mathrm{O}\right.$ in $\mathrm{CH}_{2} \mathrm{Cl}_{2}$ ), the desired compound was isolated as a white solid: run $1,105 \mathrm{mg}$ ( $81 \%$; $81 \%$ ee); run 2, $96.0 \mathrm{mg}(74 \%$; 83\% ee). The ee was determined on an AD-H column (hexanes/iso-propanol 95:5, flow $1.0 \mathrm{~mL} / \mathrm{min}$ ) with $\mathrm{t}_{\mathrm{r}}$ (minor) 16.2 min, $\mathrm{t}_{\mathrm{r}}$ (major) $18.0 \mathrm{~min}$.

mp: $103-104{ }^{\circ} \mathrm{C}$;

$[\alpha]^{20}=-93\left(\mathrm{c}=1.20, \mathrm{CH}_{2} \mathrm{Cl}_{2}\right) ; 83 \%$ ee, from $(+)-\mathrm{bpy}^{*}$;

${ }^{1} \mathrm{H}$ NMR $\left(\mathrm{CDCl}_{3}, 300 \mathrm{MHz}\right) \delta 7.62(\mathrm{~d}, J=8.1 \mathrm{~Hz}, 2 \mathrm{H}), 7.51(\mathrm{~d}, J=8.1 \mathrm{~Hz}, 2 \mathrm{H}), 7.36(\mathrm{br}$ app s, $5 \mathrm{H}), 6.06(\mathrm{~d}, J=6.6 \mathrm{~Hz}, 1 \mathrm{H}), 5.33(\mathrm{~d}, J=6.9 \mathrm{~Hz}, 1 \mathrm{H}), 5.10(\mathrm{dt}, J=11.4 \mathrm{~Hz}, 12.3 \mathrm{~Hz}$, $2 \mathrm{H}), 1.40$ (s, 9H);

${ }^{13} \mathrm{C} \mathrm{NMR}\left(\mathrm{CDCl}_{3}, 75.5 \mathrm{MHz}\right) \delta$ 169.1, 155.5, 141.7, 136.2, $130.6\left(\mathrm{q},{ }^{2} J_{\mathrm{CF}}=32.5 \mathrm{~Hz}\right), 128.7$, $128.5,128.4,127.5,125.9\left(\mathrm{q},{ }^{3} J_{\mathrm{CF}}=3.9 \mathrm{~Hz}\right), 122.3,83.6,67.3,58.2,27.9$;

IR (film) 3055, 2987, 2306, 1719, 1422, 1266, 1167, 896, 740, $705 \mathrm{~cm}^{-1}$;

HRMS (ESI) calcd for $\mathrm{C}_{21} \mathrm{H}_{22} \mathrm{~F}_{3} \mathrm{NNaO}_{4}\left(\mathrm{M}+\mathrm{Na}^{+}\right)$432.1393, found 432.1397. 


\section{Determination of Absolute Configuration}

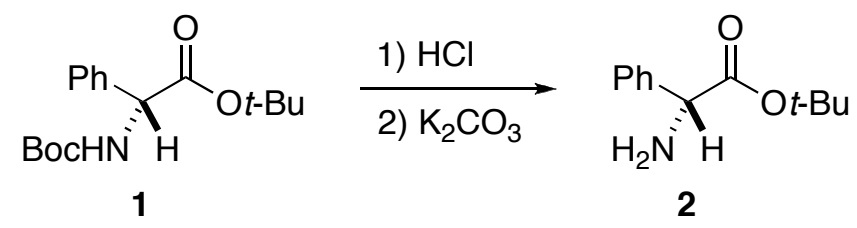

A solution of $\mathrm{HCl}$ in dioxane $(4.0 \mathrm{M} ; 0.12 \mathrm{~mL})$ was added to a solution of 1 (53.0 mg, $0.172 \mathrm{mmol} ; 94 \%$ ee; from $\left.(+)-\mathrm{bpy}^{*}\right)$ in ethyl acetate $(0.5 \mathrm{~mL})$. The mixture was stirred at room temperature for $2 \mathrm{~h}$, and then the solvent was removed. Diethyl ether $(2.0 \mathrm{~mL})$ and $10 \% \mathrm{~K}_{2} \mathrm{CO}_{3}(1.0 \mathrm{~mL})$ were added to the white residue, and the reaction mixture was stirred until all of the solids had dissolved. The product was extracted with $\mathrm{Et}_{2} \mathrm{O}$ (1.0 $\mathrm{mL} \times 2$ ), and the combined organic layers were washed with brine and then dried over $\mathrm{MgSO}_{4}$. After chromatography on silica gel ( $5 \% \mathrm{MeOH}$ in EtOAc), product 2 was isolated as a crystalline colorless solid $(30.3 \mathrm{mg} ; 85 \%)$.

$[\alpha]^{20}{ }_{\mathrm{D}}=-98\left(\mathrm{c}=1.61, \mathrm{CHCl}_{3}\right)$;

Literature value for 2: $[\alpha]^{20}{ }_{\mathrm{D}}=-108\left(\mathrm{c}=1.61, \mathrm{CHCl}_{3}\right){ }^{3}$

${ }^{1} \mathrm{H}$ NMR $\left(\mathrm{CDCl}_{3}, 500 \mathrm{MHz}\right)$ \& 7.38-7.27 (m, 5H), 4.49 (s, 1H), $1.92(\mathrm{~s}, 2 \mathrm{H}), 1.40$ (s, 9H);

${ }^{13} \mathrm{C} \mathrm{NMR}\left(\mathrm{CDCl}_{3}, 126 \mathrm{MHz}\right) \delta$ 173.4, 141.1, 128.8, 127.9, 126.8, 81.7, 59.5, 28.1.<smiles>CCCOC(=O)C(NC(=O)OCc1ccccc1)c1ccccc1</smiles>

3

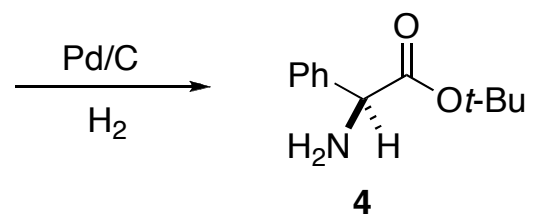

4

$\mathrm{Pd} / \mathrm{C}(50.0 \mathrm{mg})$ was added to a vial that contained 3 (50.0 mg, $0.146 \mathrm{mmol}$; $94 \%$ ee; from $(-)-$ bpy $\left.^{*}\right)$ in methanol $(0.6 \mathrm{~mL})$, and the resulting mixture was stirred under $\mathrm{H}_{2}(1$ atm) for $1 \mathrm{~h}$ at r.t. Then, the mixture was filtered through a plug of Celite. Water $(1 \mathrm{~mL})$ was added to the filtrate, and the product was extracted with $\mathrm{Et}_{2} \mathrm{O}(1 \mathrm{~mL} \times 3)$. The combined organic layers were dried over $\mathrm{MgSO}_{4}$, and the solvent was removed, thereby providing 4 as a colorless crystalline residue (30.0 mg; $99 \%$ ).

$[\alpha]^{20}{ }_{\mathrm{D}}=+95.5\left(\mathrm{c}=1.61, \mathrm{CHCl}_{3}\right)$;

Literature value for the enantiomer of $4:[\alpha]^{20}{ }_{D}=-108\left(\mathrm{c}=1.61, \mathrm{CHCl}_{3}\right){ }^{3}$ 


\section{References}

(1) Rios, R.; Liang, J.; Lo, M. M.-C.; Fu, G. C. Chem. Commun. 2000, 377-378.

(2) Maier, T. C.; Fu, G. C. J. Am. Chem. Soc. 2006, 128, 4594-4595.

(3) Smith, A. B., III; Yager, K. M.; Taylor, C. M. J. Am. Chem. Soc. 1995, 117, 1087910888. 

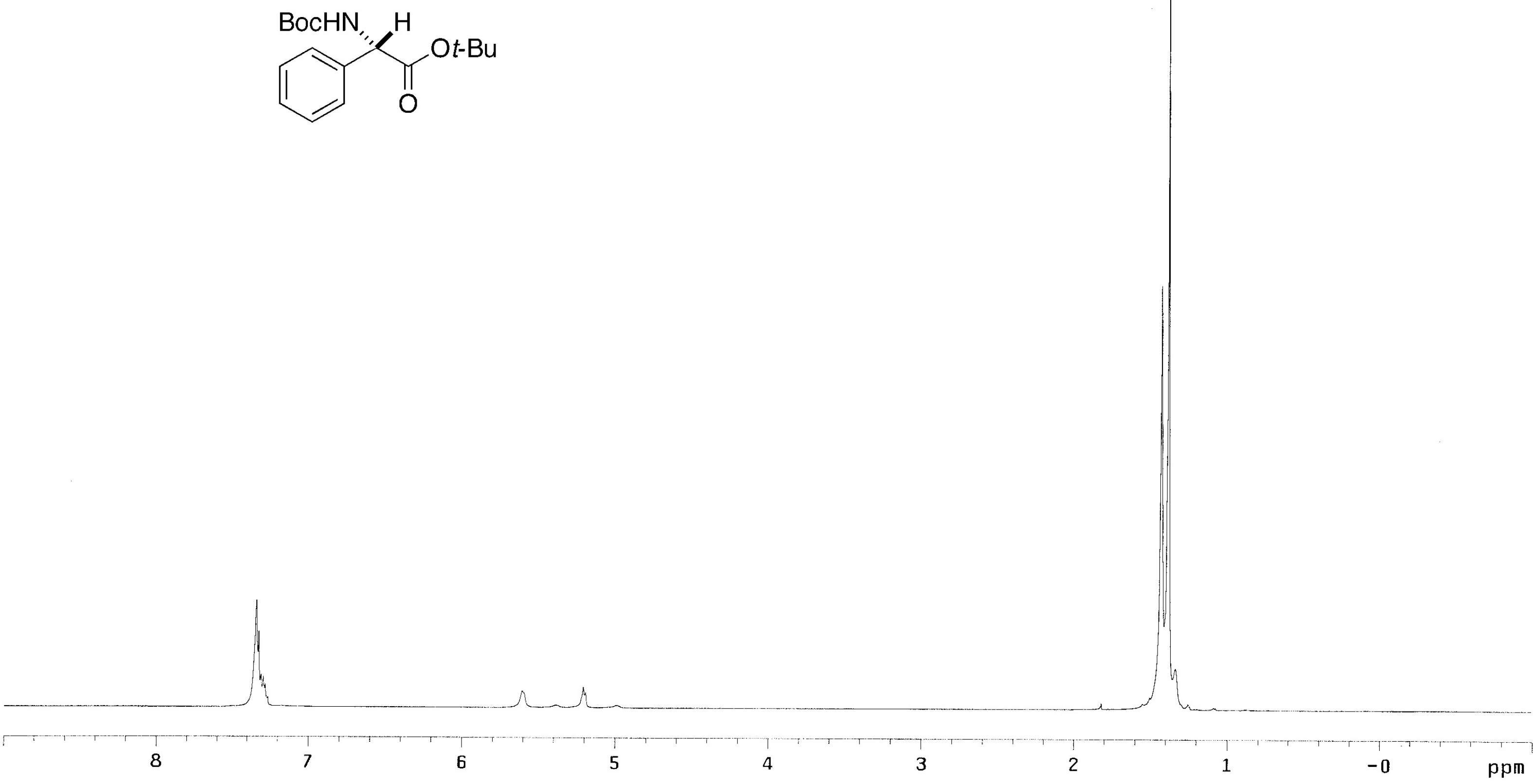

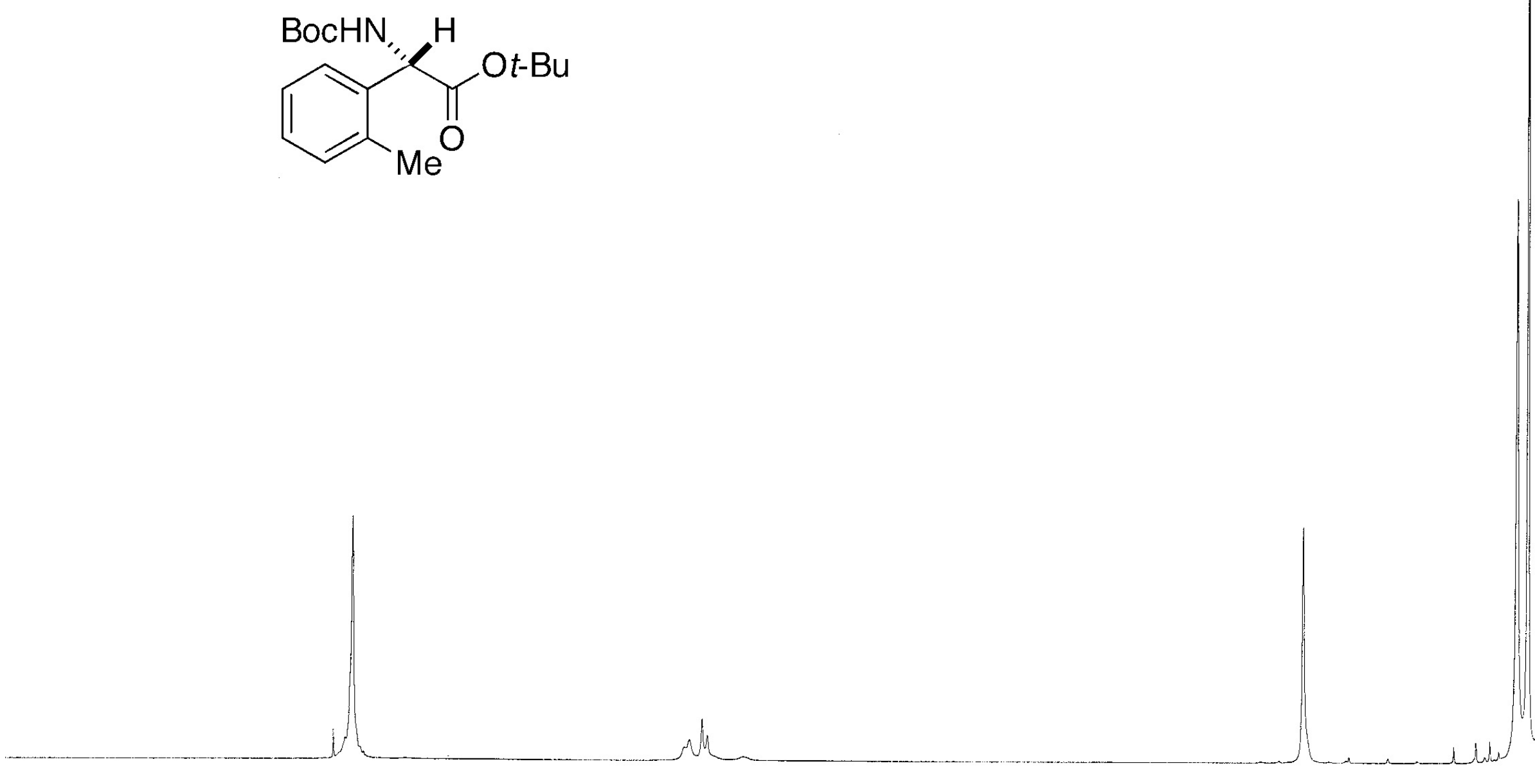


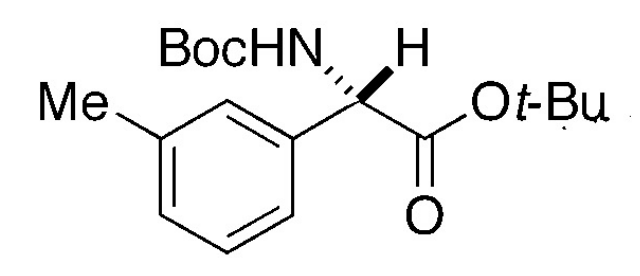


BocHN,<smiles>CCC(C)OC(=O)[C@H](NC(=O)c1ccccc1)c1ccc(OC)cc1</smiles>

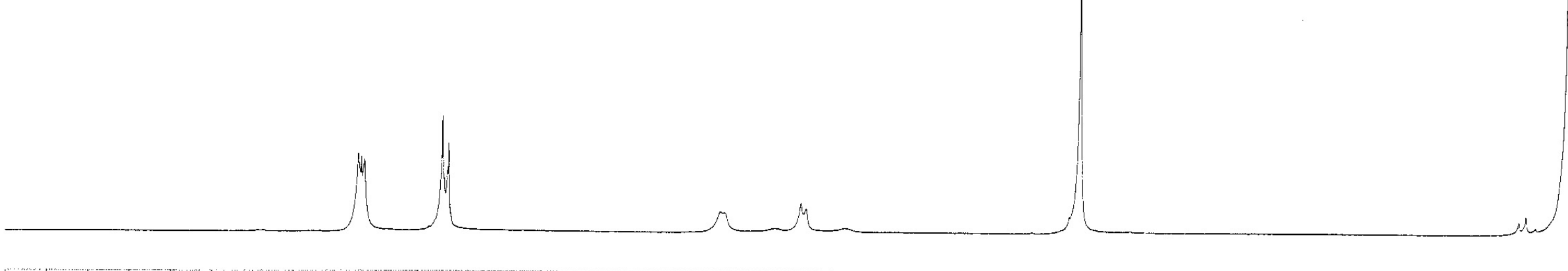




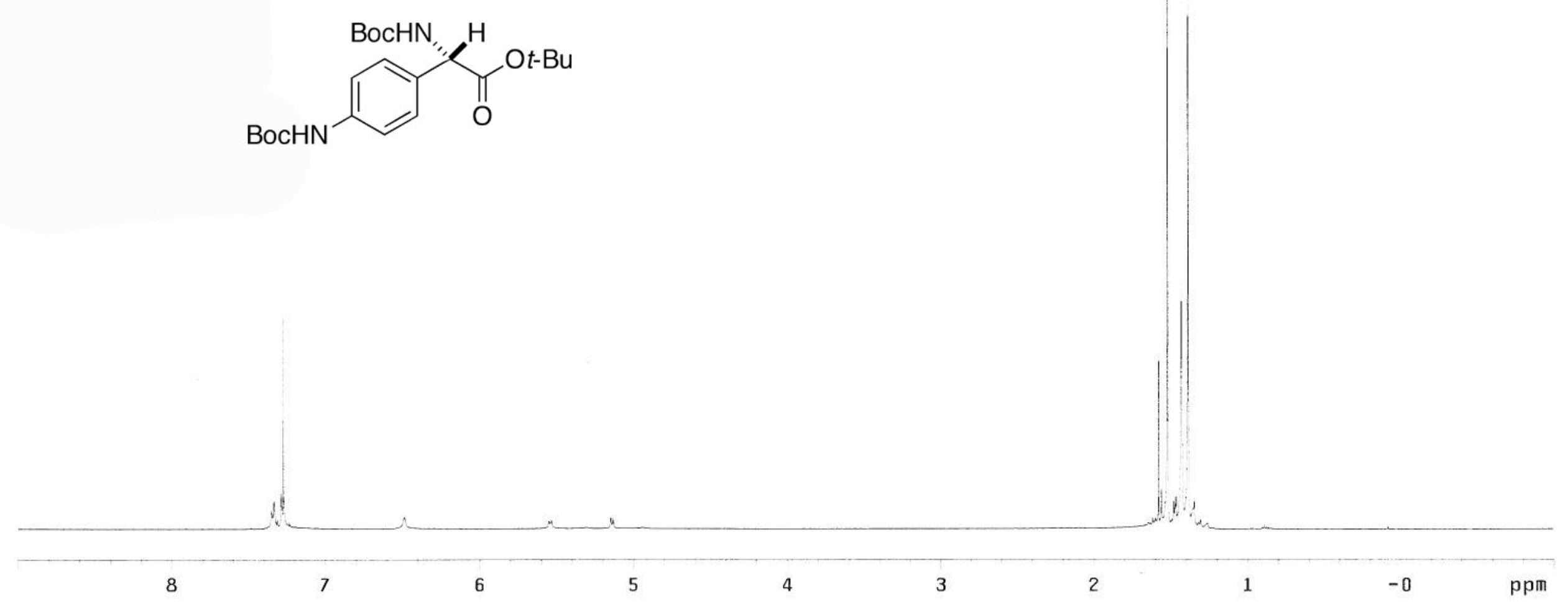



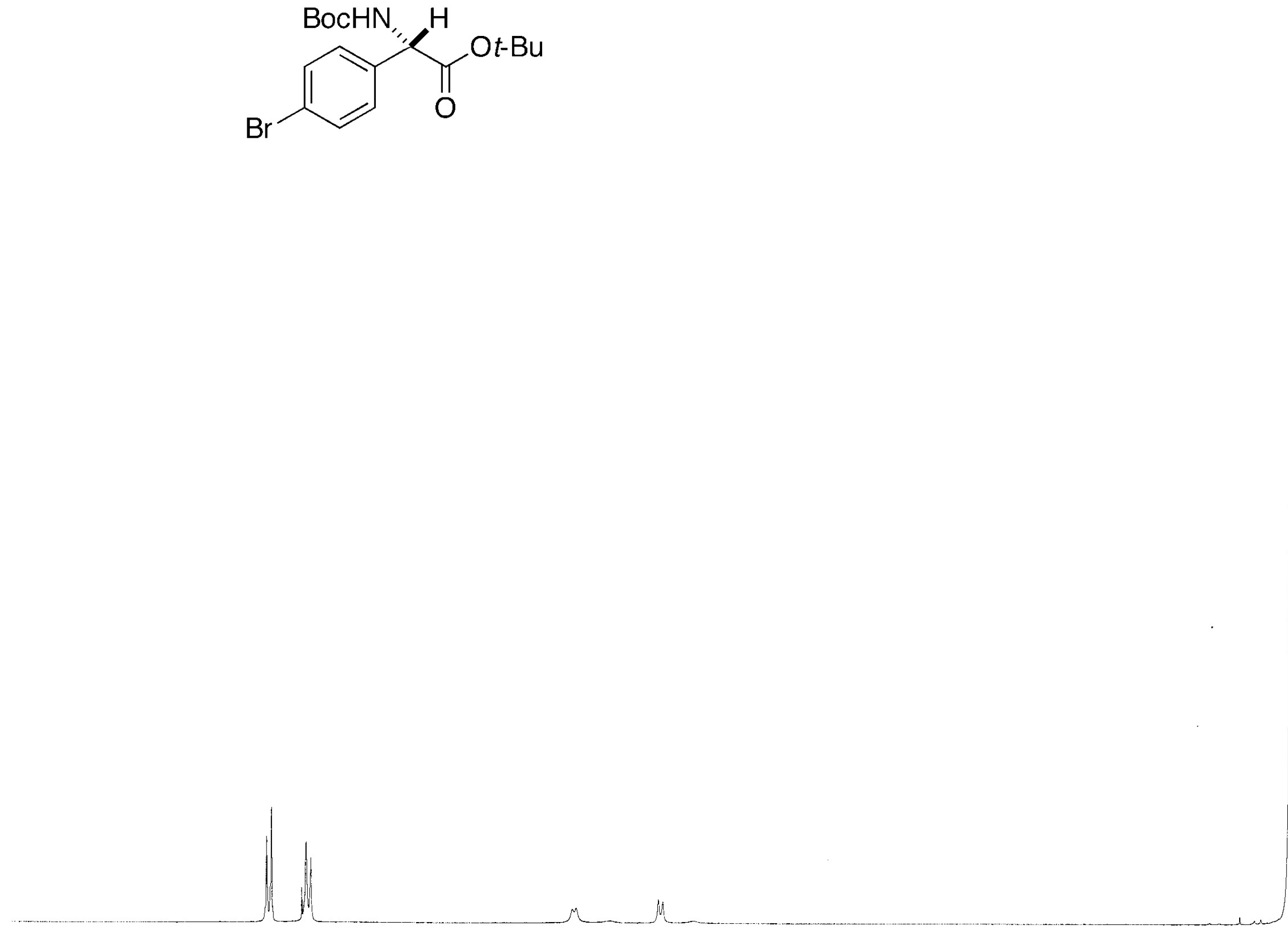

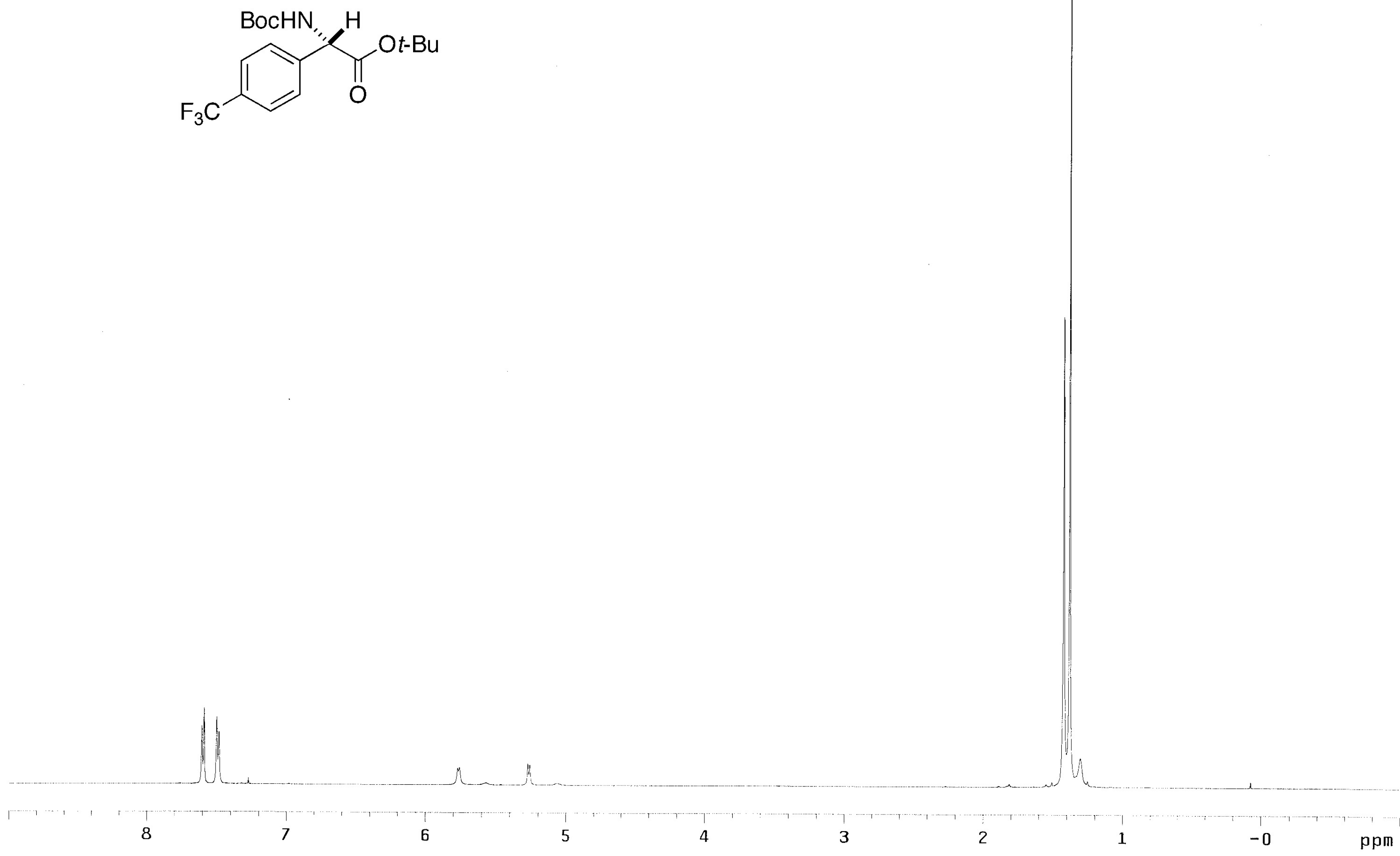

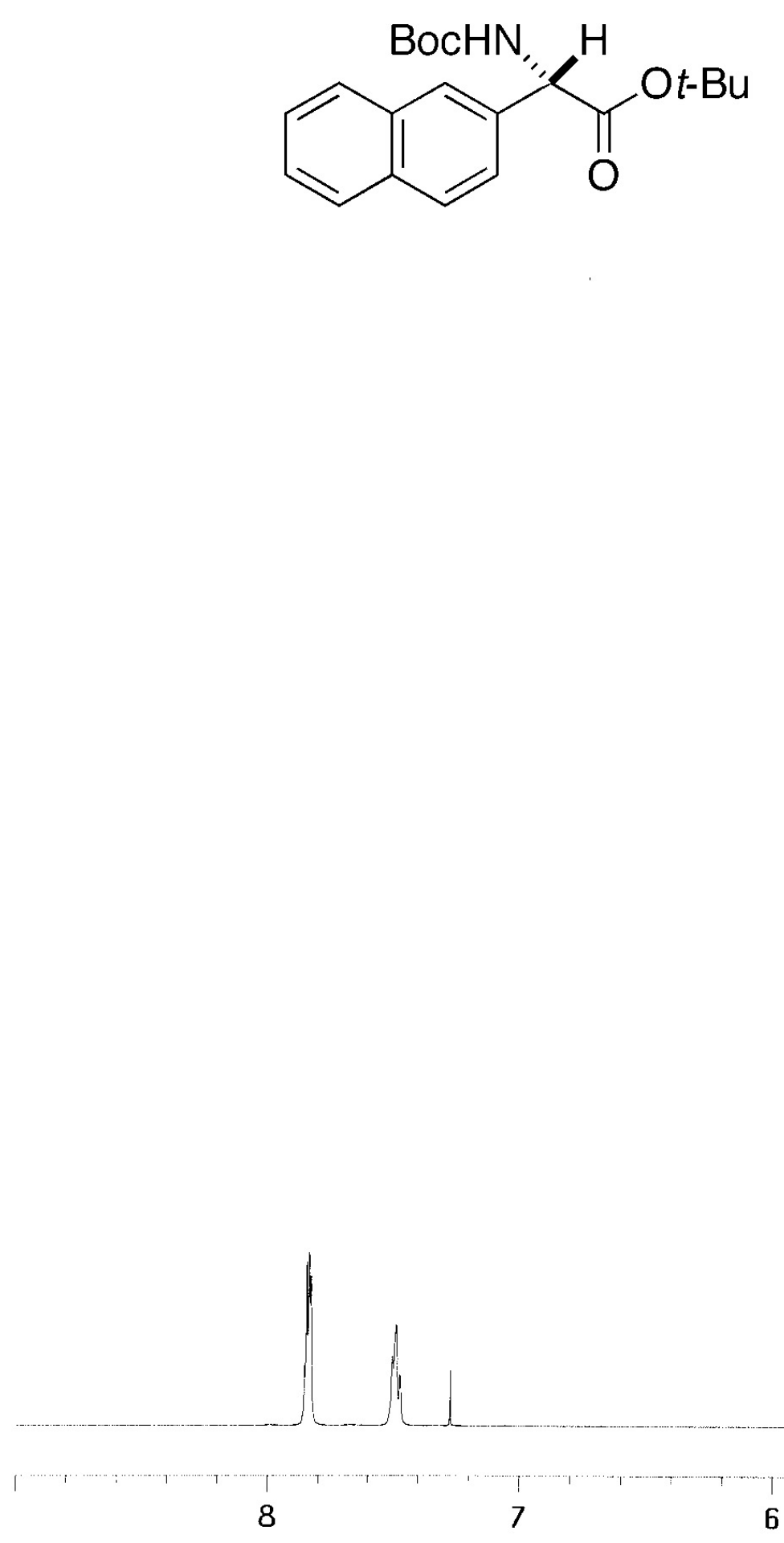

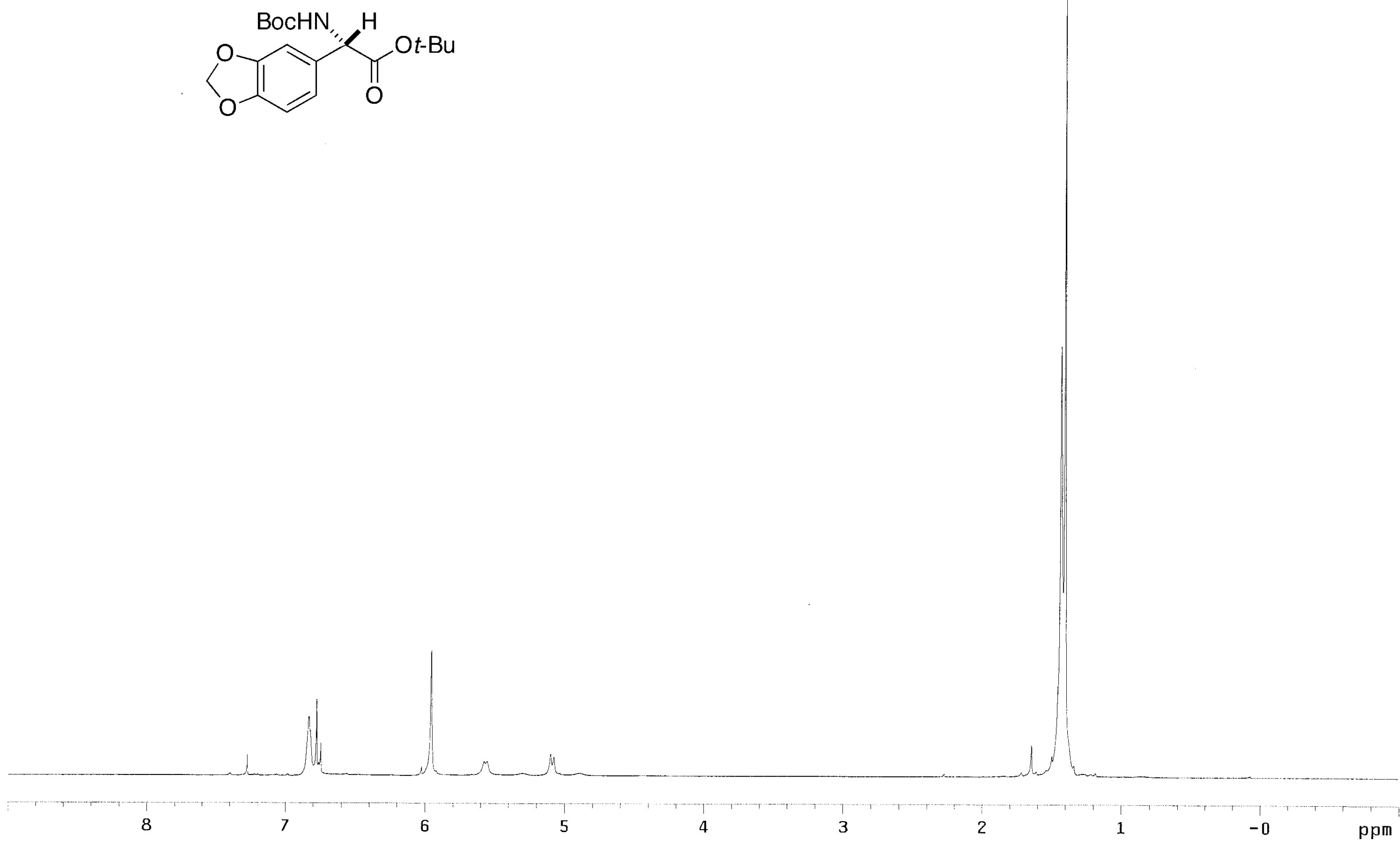

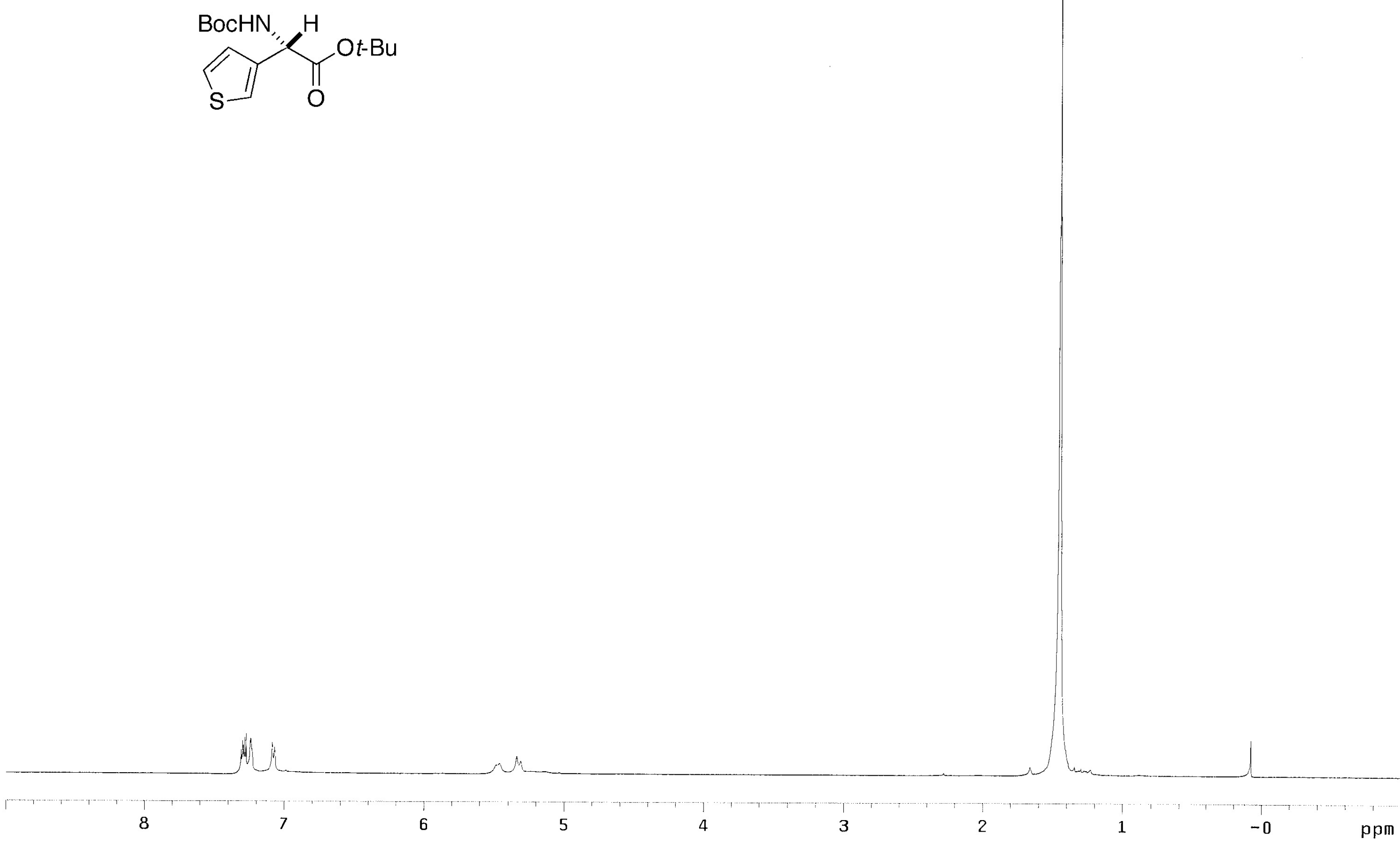
Ot-Bu

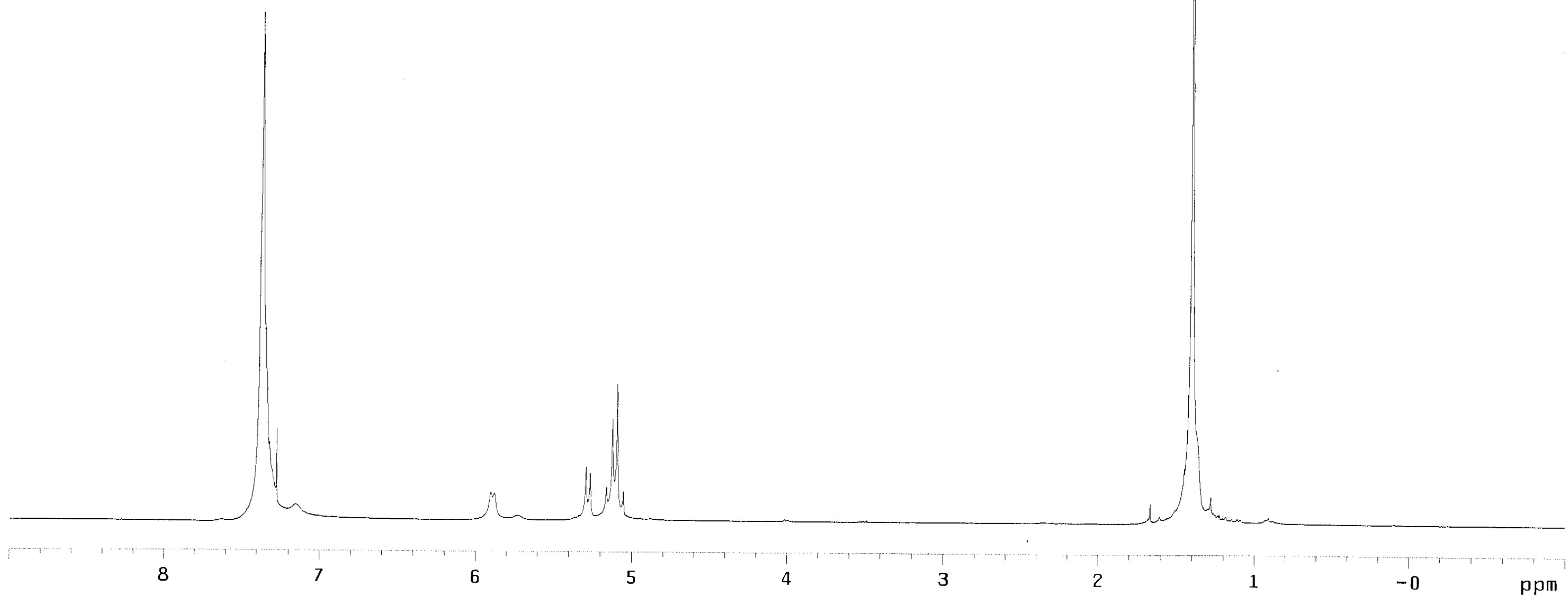



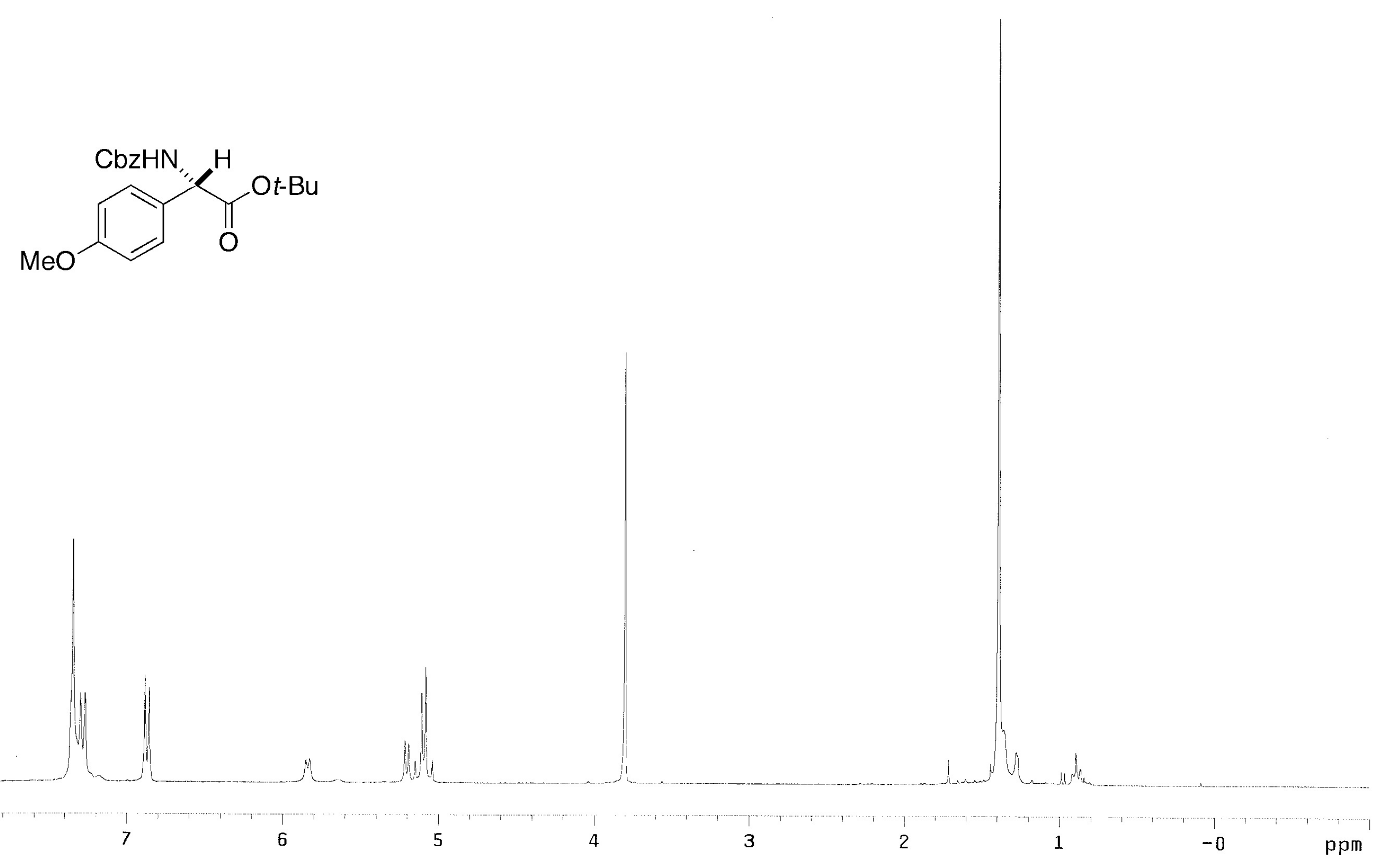

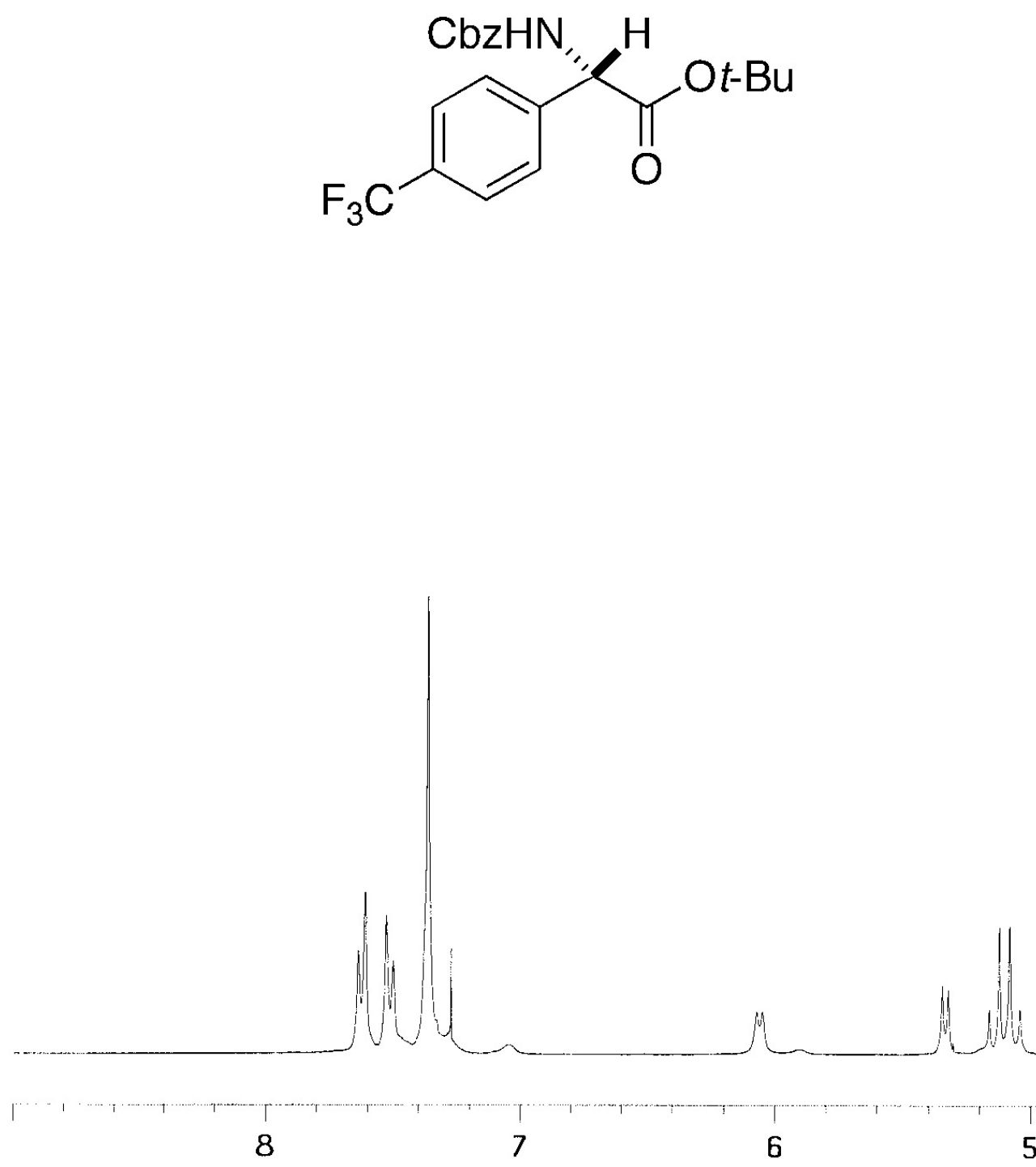
$\overbrace{\mathrm{O}}^{\mathrm{O}} \mathrm{Ot}-\mathrm{Bu}$

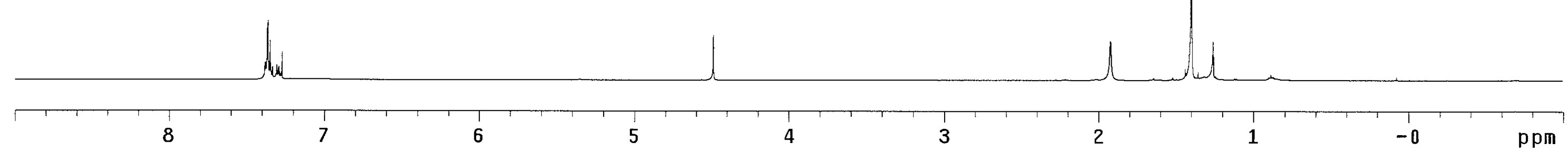

